\title{
Relationship between Lymph Node Metastasis and a Low 18F-FDG PET/CT Axillary SUVmax Value in Breast Cancer
}

\author{
Ozgur Kulahci ${ }^{1}$, Oktay Irkorucu², Emel Kocyigit Deveci ${ }^{3}$ and Zeynel Abidin Tas ${ }^{1}$ \\ ${ }^{1}$ Department of Surgery, University of Sharjah, College of Medicine, Clinical Sciences, Sharjah, UAE \\ ${ }^{2}$ Department of Nuclear Medicine, University of Health Sciences, Adana City Education and Research Hospital, Turkey
}

\begin{abstract}
Objective: To evaluate breast cancer patients with low F18-fluorodeoxy-glucose positron emission tomography/computed tomography scan (FPET) of axilla standardised maximum uptake value (SUVmax) along with their histopathological findings regarding axillary lymph node metastasis (LNM).

Study Design: A descriptive study.

Place and Duration of the Study: Adana City Education and Research Hospital, Turkey, from January 2015 to October 2018.

Methodology: The current study analysed the findings of 113 patients according to age, histopathological axillary LNM, estrogen and progesterone receptor status, tumour size, histological grade, Ki-67, HER2 and preoperative FPET SUVmax findings. Histopathological and immunohistochemical comparisons were made between FPET axilla SUVmax and univariate and multivariate parameters in breast carcinoma in terms of LNM.

Results: The authors analysed the receiver operator characteristic curve for the FPET axilla SUVmax and set the cut-off value to 1.84 to predict LNM. However, it was also found that the SUVmax detected metastases in the axilla at values lower than 1.84. In the multivariate analysis, a statistically significant relationship was found between axilla LNM cases that have FPET axilla SUVmax less than 1.84, a Ki-67 index greater than $15 \%$ and tumour size greater than $2 \mathrm{~cm}(\mathrm{p}<0.05)$.

Conclusion: Values that were above the axilla SUVmax limit of 1.84 in FPET helped determine LNM. However, further evaluation of patients is needed comparing axilla SUVmax of less than 1.84 in FPET together with the Ki-67 proliferation index and tumour size.
\end{abstract}

Key Words: Breast, Cancer, Lymph nodes, Metastasis, PET, Pathology.

How to cite this article: Kulahci O, Irkorucu O, Deveci EK, Tas ZA. Relationship between Lymph Node Metastasis and a Low 18F-FDG PET/CT Axillary SUVmax Value in Breast Cancer. J Coll Physicians Surg Pak 2021; 31(05):511-516.

\section{INTRODUCTION}

Breast cancer is the most common female cancer globally. ${ }^{1}$ The absence of axillary LNM is the most critical prognostic survival factor for breast cancer patients. ${ }^{2}$ Other prognostic factors, such as tumour size, histopathological grade, and estrogen receptor (ER) status can be determined using imaging methods, histopathological examination and immunohistochemical staining, respectively. Axillary lymph node metastases can also be determined with radiology and histopathology. For early-stage breast cancer, the most accepted method for detecting LNM is surgical axillary lymph node dissection (ALND), sentinel lymph node biopsy (SLNB). ${ }^{3}$

Correspondence to: Dr. Ozgur Kulahci, Department of Pathology, University of Health Sciences, Adana City Education and Research Hospital, Turkey

E-mail: ozgurkulahci@hotmail.com

Received: July 01, 2020; Revised: March 25, 2021;

Accepted: April 15, 2021

DOI: https://doi.org/10.29271/jcpsp.2021.05.511

Sophisticated imaging methods such as breast magnetic reso- nance imaging, ultrasonography, and F18-fluorodeoxy-glucose positron emission tomography combined with CT (FPET) are also used preoperatively to identify axillary LNM. FPET is recommended for advanced breast carcinoma and is influential in determining axillary LNM, especially if the cancer is in an advanced regional stage or a distant metastatic state. ${ }^{4}$ FPET has been reported to have different specificity and sensitivity values in various studies. ${ }^{5-10}$

While increased axilla FPET standardised maximum-uptake-value (SUVmax) is a valuable measure of LNM, tumour metastasis can still be seen in patients with a lower SUVmax.

The objective of this study was to analyse the relationship between low SUVmax and the histopathological findings of tumourmetastasis.

\section{METHODOLOGY}

The study included 113 cases that were undergoing a preoperative FPET scan, then breast surgery, followed by pathological axillary lymph node evaluation between January 2015 and October 2018. This study evaluated histopathologically the correlation between axillary LNM and other parameters. 
All the participants were diagnosed with infiltrative ductal carcinoma by preoperative true-cut biopsy. Excluded from the study were males and patients without a preoperative FPET scan. Patients with an excisional biopsy, neoadjuvant chemotherapy, recurrent breast cancer, and breast tumours other than infiltrative ductal carcinoma axillary lymph node were also excluded.

The patients were required to fast for no less than 6 hours before obtaining standard FPET images. Average serum glucose values were confirmed as below $200 \mathrm{mg} / \mathrm{dl}$ before the ${ }^{18} \mathrm{~F}$-FDG application. A PET device containing a lithium oxyloxylicate PET scanner (Philips-Ingenuity) and a multi-slice CT scanner were used for FPET images. FPET images were acquired an hour after applying $5 \mathrm{MBq}(0.1 \mathrm{mCi}){ }^{18} \mathrm{~F}-\mathrm{FDG}$ per kilogram of body weight, and the bed positions were held for 2-4 minutes each. CT scans were performed using oral contrast material and were utilised for attenuation correction of PET images and anatomic lesion localisation and diagnostics. FPET image analyses were carried out by a single-blind observer who was unaware of the histologic typeand tumourgrade. FPETtests were quantitatively and qualitatively analysed. The single maximum pixel value of the lymph nodes on the same side as the tumour (SUVmax) was identified along with the slice exhibiting the highest radioactivity concentration.

An experienced oncological surgeon operated on patients with a primary tumour. Evaluationswere then performed on the intraoperative biopsy of the sentinel lymph node or the postoperative dissection of the axillary lymph node, or both, including surgical resection. Serial and immunohistochemical staining was performed on the lymph node where routine histopathological methods could not detect metastasis.

These preparations were evaluated by the pathologists among the study's authors who were experienced with histopathological and immunohistochemical assessments. Age, histopathological axillary LNM, tumour size, histological grade, ER, PR, Ki-67, HER2 and preoperative FPET SUVmax findings were analysed. Each participant's age was entered in years. The histopathological axillary LNM was recorded as positive or negative. Tumour diameter was classified as greater than or less than $2 \mathrm{~cm}$. The histological grade (according to the Nottingham histological score) was recorded as grade 1, 2 or 3. According to the results of immunohistochemical staining, ER (BOND ${ }^{\mathrm{TM}}$ ER Clone 6-F11 Primary Antibody, Leica Biosystems, UK.) and PR (BOND ${ }^{\mathrm{TM}}$, Primary Antibody Progesterone Receptor, Leica Biosystems, UK. [16]) were classified as positive, if greater than $1 \%$ and classified as negative if less than $1 \%$. Immunohistochemical staining was considered positive, if the HER2 (BOND ${ }^{\mathrm{TM}}$, Primary Antibody cerbB-2 Oncoprotein, Leica Biosystems, UK.) receptor was stained $3+$ and was treated with HER2/neu gene amplification by fluorescent in situ hybridisation in cases where the HER2 receptor was stained $2+$. The Ki-67 value (Novocastra ${ }^{\mathrm{TM}}$, Liquid Mouse Monoclonal Antibody Ki-67 Antigen, Leica Biosystems, UK.) was recorded as greater than or less than $15 \%$. The numer- ical value of the FPET axillary lymph node SUVmax was recorded. Then the preoperative FPET axillary lymph node SUVmax value and histopathological axillary metastasis were compared with the above pathological findings.

SPSS version 19 package software (SPSS Inc., Chicago, Illinois, USA) was used for the statistical analysis. The categorical variables, including tumour size, axillary lymph node, and histological grade, are summarised as number $(n)$ and percentage (\%). Descriptive statistics for the continuous variable of age and the FPET axilla lymph node SUV-max variable are summarised as the mean \pm standard deviation. The cut-off value for FPET axilla SUVmax for predicting axillary LNM were determined by analysing the ROC curve. The Chi-squared test, Fisher's Exact test or the likelihood ratio test was used for analysing categorical variables. Sensitivity, specificity, positive predictive value, and also negative predictive value were used to investigate the accuracy of the diagnostic tests regarding the FPET and axillary lymph nodes. Avalue of $p<0.05$ was considered statistically significant.

\section{RESULTS}

The patients were between the ages of 30-86 (mean $52.42 \pm$ 11.66 years). The value of the FPET axilla SUVmax ranged from 0-38.24 (mean $1.80 \pm 4.47$ ). For predicting axillary LNM, FPET axilla SUVmax receiver operator characteristic curve analysis was examined. The area under the curve was $75.6 \%$, and the confidence interval was 66.3-84.8\%. The cut-off value for FPET axilla SUVmax was set at 1.84, while the sensitivity was $53.1 \%$, the specificity was $85.9 \%$, and the likelihood ratio was 3.8 .

Analysis of univariate parameters revealed a statistically significant relationship between histopathological axillary LNM and tumour size, histological grade, Ki-67 and the FPET axilla SUVmax cut-off value ( $p<0.05$ ).

The FPET values greater than and less than the established cutoff value of 1.84 were then compared. Our analysis of univariate parameters found a statistically significant correlation between the FPET axilla SUVmax cut-off value and the histological grade and Ki-67 ( $p<0.05$ ). Axilla SUVmax cut-off values above 1.84 correlated with high Ki-67 proliferation indicesand high histological grades (Tablel).

While the axillary LNM was 49 (43.4\%) in the histopathological examination, the FPET axillary lymph node was 34 (30.1\%) for the group having a SUVmax value greater than 1.84. There was a 13.3\% difference between FPET axillary lymph node SUV-max cut-off value 1.84 and the histopathological axilla LNM.

This study also employed multivariable parameters in examining the groups above and below the FPET axillary lymph node SUVmax cut-off value of 1.84. In these examinations, a significant correlation was observed for the Ki-67 proliferation index, tumour size and histopathological axillary LNM in cases with an FPET axillary lymph node SUVmax less than 1.84 ( $p$ $<0.05$ ).

Table I: Correlation between FPET axillary lymph node SUVmax cut-off value and histopathological and immunohistochemical findings in the univariate analysis of different parameters. 


\begin{tabular}{|c|c|c|c|}
\hline & $\begin{array}{c}\text { FPET axillary lymph } \\
\text { node SUVmax }<1.84 \\
n(\%)\end{array}$ & $\begin{array}{c}\text { FPET axillary lymph } \\
\text { node SUVmax }>1.84 \\
n(\%)\end{array}$ & p-value \\
\hline $\begin{array}{l}\text { Tumour size } \\
\quad \leq 2 \mathrm{~cm} \\
>2 \mathrm{~cm}\end{array}$ & $\begin{array}{l}36(78.3) \\
43(64.2)\end{array}$ & $\begin{array}{l}10(21.7) \\
24(35.8)\end{array}$ & 0.109 \\
\hline $\begin{array}{l}\text { Histological grade } \\
\text { G1 } \\
\text { G2 } \\
\text { G3 }\end{array}$ & $\begin{array}{l}14(82.4) \\
56(75.7) \\
9(40.9)\end{array}$ & $\begin{array}{c}3(17.6) \\
18(24.3) \\
13(59.1)\end{array}$ & 0.004 \\
\hline $\begin{array}{l}\text { ER } \\
\text { Negative } \\
\text { Positive }\end{array}$ & $\begin{array}{l}7(53.8) \\
72(72)\end{array}$ & $\begin{array}{l}6(46.2) \\
28(28)\end{array}$ & 0.206 \\
\hline $\begin{array}{l}\text { PR } \\
\text { Negative } \\
\text { Positive }\end{array}$ & $\begin{array}{l}10(55.6) \\
69(72.6)\end{array}$ & $\begin{array}{c}8(44.4) \\
26(27.4)\end{array}$ & 0.148 \\
\hline $\begin{array}{l}\text { HER2 } \\
\text { Negative } \\
\text { Positive }\end{array}$ & $\begin{array}{l}58(75.3) \\
21(58.3)\end{array}$ & $\begin{array}{l}19(24.7) \\
15(41.7)\end{array}$ & 0.067 \\
\hline $\begin{array}{l}\text { Ki-67 } \\
\quad \leq 15 \% \\
>15 \%\end{array}$ & $\begin{array}{l}42(87.5) \\
37(56.9)\end{array}$ & $\begin{array}{c}6(12.5) \\
28(43.1)\end{array}$ & $<0.001$ \\
\hline $\begin{array}{l}\text { HPALNM } \\
\text { Negative } \\
\text { Positive }\end{array}$ & $\begin{array}{c}55(85.9) \\
24(49)\end{array}$ & $\begin{array}{l}9(14.1) \\
25(51)\end{array}$ & $<0.001$ \\
\hline
\end{tabular}

Table II: FPET axilla lymph node SUVmax cut-off value in multivariable parameter analysis and its relationship to categorical variables with axillary LNM.

\begin{tabular}{|c|c|c|c|c|}
\hline $\begin{array}{l}\text { FPET } \\
\text { axillary lymph node } \\
\text { SUVmax }\end{array}$ & $\begin{array}{l}\text { Histopathological and } \\
\text { immunohistochemical findings }\end{array}$ & $\begin{array}{c}\text { Histopathological } \\
\text { axillary lymph node } \\
\text { metastasis negative } \\
\text { n (\%) }\end{array}$ & \begin{tabular}{|c|} 
Histopathological \\
axillary lymph node metastasis \\
positive \\
n (\%)
\end{tabular} & p-value \\
\hline$<1.84$ & $\begin{array}{l}\text { Ki- } 67 \leq 15 \% \\
\text { Ki- } 67>15 \%\end{array}$ & $\begin{array}{l}35(83.3) \\
20(54.1)\end{array}$ & $\begin{array}{c}7(16.7) \\
17(45.9) \\
\end{array}$ & 0.005 \\
\hline$>1.84$ & $\begin{array}{l}\mathrm{Ki}-67 \leq 15 \% \\
\mathrm{Ki}-67>15 \%\end{array}$ & $\begin{array}{l}5(83.3) \\
4(14.3)\end{array}$ & $\begin{array}{c}1(16.7) \\
24(85.7)\end{array}$ & 0.001 \\
\hline$<1.84$ & $\begin{array}{l}\mathrm{TS} \leq 2 \mathrm{~cm} \\
\mathrm{TS}>2 \mathrm{~cm}\end{array}$ & $\begin{array}{l}32(88.9) \\
23(53.5)\end{array}$ & $\begin{array}{c}4(11.1) \\
20(46.5)\end{array}$ & 0.001 \\
\hline$>1.84$ & $\begin{array}{l}\mathrm{TS} \leq 2 \mathrm{~cm} \\
\mathrm{TS}>2 \mathrm{~cm}\end{array}$ & $\begin{array}{c}4(40) \\
5(20.8)\end{array}$ & $\begin{array}{c}6(60) \\
19(79.2)\end{array}$ & 0.248 \\
\hline$<1.84$ & $\begin{array}{l}\text { G1 } \\
\text { G2 } \\
\text { G3 }\end{array}$ & $\begin{array}{c}11(78.6) \\
39(69.6) \\
5(55.6)\end{array}$ & $\begin{array}{c}3(21.4) \\
17(30.4) \\
4(44.4)\end{array}$ & 0.504 \\
\hline$>1.84$ & $\begin{array}{l}\mathrm{G} 1 \\
\mathrm{G} 2 \\
\mathrm{G} 3\end{array}$ & $\begin{array}{l}2(66.7) \\
5(27.8) \\
2(15.4)\end{array}$ & $\begin{array}{c}1(33.3) \\
13(72.2) \\
11(84.6)\end{array}$ & 0.218 \\
\hline$<1.84$ & $\begin{array}{l}\text { ER }(-) \\
\operatorname{ER}(+)\end{array}$ & $\begin{array}{c}6(85.7) \\
49(68.1)\end{array}$ & $\begin{array}{c}1(14.3) \\
23(31.9)\end{array}$ & 0.332 \\
\hline$>1.84$ & $\begin{array}{l}\text { ER }(-) \\
\operatorname{ER}(+)\end{array}$ & $\begin{array}{c}3(50) \\
6(21.4)\end{array}$ & $\begin{array}{c}3(50) \\
22(78.6)\end{array}$ & 0.306 \\
\hline$<1.84$ & $\begin{array}{l}\text { PR (-) } \\
\text { PR }(+)\end{array}$ & $\begin{array}{c}8(80) \\
47(68.1)\end{array}$ & $\begin{array}{c}2(20) \\
22(31.9)\end{array}$ & 0.445 \\
\hline$>1.84$ & $\begin{array}{l}\text { PR (-) } \\
\text { PR }(+)\end{array}$ & $\begin{array}{c}4(50) \\
5(19.2)\end{array}$ & $\begin{array}{c}4(50) \\
21(80.8)\end{array}$ & 0.165 \\
\hline$<1.84$ & $\begin{array}{l}\text { HER2 }(-) \\
\text { HER2 }(+)\end{array}$ & $\begin{array}{c}40(69) \\
15(71.4)\end{array}$ & $\begin{array}{l}18(31) \\
6(28.6)\end{array}$ & 0.833 \\
\hline$>1.84$ & $\begin{array}{l}\operatorname{HER} 2(-) \\
\operatorname{HER} 2(+)\end{array}$ & $\begin{array}{l}8(42.1) \\
1(6.7)\end{array}$ & $\begin{array}{l}11(57.9) \\
14(93.3)\end{array}$ & 0.020 \\
\hline
\end{tabular}

Table III: FPET axillary lymph node SUVmax cut-off value and its relationship with axillary LNM when categorical variables are used together in multivariate analysis.

\begin{tabular}{|l|l|l|c|c|}
\hline FPET axillary lymph node SUVmax & Ki-67 & $\begin{array}{c}\text { Tumour } \\
\text { size }\end{array}$ & $\begin{array}{c}\text { Histopathological } \\
\text { axillary lymph } \\
\text { node metastasis } \\
\text { negative } \\
\text { n(\%) }\end{array}$ & $\begin{array}{c}\text { Histopathological } \\
\text { axillary lymph } \\
\text { node metastasis } \\
\text { positive } \\
n(\%)\end{array}$ \\
\hline
\end{tabular}




\begin{tabular}{|c|c|c|c|c|c|}
\hline \multirow{2}{*}{$<1.84$} & $\leq 15 \%$ & $\begin{array}{l}\leq 2 \mathrm{~cm} \\
>2 \mathrm{~cm}\end{array}$ & $\begin{array}{c}23(92) \\
12(70.6)\end{array}$ & $\begin{array}{c}2(8) \\
5(29.4)\end{array}$ & 0.068 \\
\hline & $>15 \%$ & $\begin{array}{l}\leq 2 \mathrm{~cm} \\
>2 \mathrm{~cm}\end{array}$ & $\begin{array}{c}9(81.8) \\
11(42.3)\end{array}$ & $\begin{array}{c}2(18.2) \\
15(57.7)\end{array}$ & 0.036 \\
\hline \multirow{2}{*}{$>1.84$} & $\leq 15 \%$ & $\begin{array}{l}\leq 2 \mathrm{~cm} \\
>2 \mathrm{~cm}\end{array}$ & $\begin{array}{c}3(75) \\
2(100)\end{array}$ & $\begin{array}{l}1(25) \\
0(0)\end{array}$ & 0.439 \\
\hline & $>15 \%$ & $\begin{array}{l}\leq 2 \mathrm{~cm} \\
>2 \mathrm{~cm}\end{array}$ & $\begin{array}{l}1(16.7) \\
3(13.6)\end{array}$ & $\begin{array}{c}5(83.3) \\
19(86.4)\end{array}$ & $>0.999$ \\
\hline
\end{tabular}

Axillary LNM was detected in $45.9 \%$ of the participants with a Ki-67 proliferation index above $15 \%$ and $46.5 \%$ of the participants with tumour size greater than $2 \mathrm{~cm}$ (Table II).

An evaluation was also performed of the multivariate parameters of axillary lymph node SUVmax less than 1.84, Ki-67 proliferation index greater than $15 \%$ and tumour size greater than $2 \mathrm{~cm}$ in FPET and LNM. The result was $57.7 \%$, and the correlation in this respect was statistically significant $[(p=0.036$, Table III $)]$.

\section{DISCUSSION}

The prognosis, treatment and management of breast carcinoma depends on T stage, histological grade, HER2, PR status, ER status, axilla LNM and the extent of metastasis. Among these factors, axillary LNM is a crucial component for survival. ${ }^{2,11}$ The gold standard of lymph node evaluation is axillary lymph node dissection. In various studies, the sensitivity of FPET in the axillary staging of breast carcinoma has been found to range from $37-70 \%$, with a specificity of $92-100 \% .^{5-10}$ In this study, the FPET axillary lymph node SUVmax cut-off value for histopathological axilla LNM had sensitivity and specificity values of $73.5 \%$ and $85.5 \%$, respectively. These results, which differ from those in the literature, may be due to the different histopathological and immunohistochemical features of the tumour. The design of the current study considered these features, establishing the FPET axillary lymph node SUVmax cut-off value at 1.84 . Researchers in similar studies established the cut-off value at 1.1 or $3.9 .^{12,13}$ The findings in this study were correlated with the pathological and immunohistochemical parameters in the primary breast tumour to determine the FPET axillary lymph node SUVmax cut-off value for LNM.

Many previous research has determined that as tumour size increases in breast carcinoma, the risk of axillary LNM also increases. ${ }^{14,15}$ In the present study, the analysis of univariate parameters determined that as tumour size increased, axillary LNM also increased histopathologically. However, in the univariate analysis, no relationship was found between tumour size and the cut-off value for FPET axilla lymph node SUVmax. Some of the previous studies did not find a relationship between histological grade and FPET axilla involvement, while others reported a correlation. ${ }^{16,17}$ The current analysis of univariate parameters found a relationship between histological grade and FPET axilla lymph node SUVmax cut-off value in terms of metastasis. High-grade tumours had a higher rate of axillary metastasis. Since high histological grade tumours are known to be more aggressive and often metastasise, the findings were as predicted. While some previous studies did not determine a relationship between $\mathrm{Ki}-67$ indices and axillary LNM, others did find a relationship, as did this study. ${ }^{18,19}$

Similar studies in the literature focused mainly on the correlation of LNM and cases with a high FPET axillary lymph node SUVmax. Similarly, the FPET axillary lymph node SUVmax values in our study helped determine LNM greater than 1.84. The current study's aim differed from previous studies by investigating if any histopathological and immunohistochemical findings affect the axillary LNM of low SUVmax patients in FPET if analysed together. In axillary LNM, the FPET axillary lymph node SUVmax cut-off value was used together with categorical variables, such as Ki-67 indices, tumour size, histological grade, ER, PR and HER2, in the analysis of multivariate parameters.

The resulting analysis firstly revealed a statistically significant relationship between histopathological axillary LNM and tumour size, histological grade, Ki-67 indices and the FPET axilla SUVmax cut-off value $(p<0.05)$. Next, when the cut-off values greater than and less than 1.84 were compared, it was found that the cut-off value for FPET axillary lymph node SUVmax was predictive of axillary LNM. There was a statistically significant correlation between the FPET axilla SUVmax cut-off value and the histological grade and Ki-67 indices ( $p$ $<0.05$ ). Axilla SUV-max cut-off values greater than 1.84 correlated with high Ki-67 indices and high histological grades. Finally, analysing multivariate parameters resulted in a significant correlation for the Ki-67 proliferation index, tumour size and histopathological axillary LNM in cases with an FPET axillary lymph node SUV-max less than 1.84 ( $p$ $<0.05$ ). The multivariate parameters were then simultaneously evaluated, comprising axillary lymph node SUV-max less than 1.84 , Ki-67 proliferation index greater than $15 \%$ and tumour size greater than $2 \mathrm{~cm}$ in FPET and LNM. The resulting correlation was significant $(p<0.05)$.

\section{CONCLUSION}

There was a statistically significant relationship between histopathological axillary LNM and the FPET axilla SUVmax cut-off values greater than and less than 1.84 .

For patients with an FPET axillary lymph node SUVmax less than 1.84, a follow up on axilla LNM will be necessary if the $\mathrm{Ki}-67$ indices is above $15 \%$ and tumour diameter is greater 
than $2 \mathrm{~cm}$. Therefore, it is suggested that breast cancer patients with a low axillary lymph node SUVmax value be evaluated together with pathological parameters. Conducting similar studies on this subject may contribute further to the literature.

\section{ETHICAL APPROVAL:}

The approval of Adana City Training and Research Hospital Ethics Committee was obtained (Decision No. 295).

\section{PATIENTS' CONSENT:}

As the study was designed retrospectively, data was collected from clinical archives after ethical approval.

\section{CONFLICT OF INTEREST:}

The authors declared no conflict of interest.

\section{AUTHORS' CONTRIBUTION:}

OK, OI, EKD, ZAT: Substantial contributions to the conception or design of the work; or the acquisition, analysis, or interpretation of data for the work. Drafting the work or revising it critically for important intellectual content; final approval of the version to be published.

\section{REFERENCES}

1. Momenimovahed Z, Salehiniya H. Epidemiological characteristics of and risk factors for breast cancer in the world. Breast Cancer (Dove Med Press) 2019; 11:151-64. doi: 10.2147/BCTT.S176070.

2. Morlino A, Torre GL, Patitucci G, Cammarota A. Axillary treatment of patients with breast cancer and micrometastatic disease in the sentinel lymph node our experience. Ann Ital Chir 2017; 88:360-4.

3. Jolinière JB, Major A, Khomsi F, Ali NB, Guillou L, Feki A. The sentinel lymph node in breast cancer: problems posed by examination during surgery. A review of current literature and management. Front Surg 2018; 5:56.

4. Groheux D, Cochet A, Humbert O, Alberini JL, Hindié E, Mankoff D. ${ }^{18} \mathrm{~F}-\mathrm{FDG}$ PET/CT for staging and restaging breast cancer. J Nucl Med 2016; 57 (Suppl 1):17S-26S. doi: 10.2967/jnumed.115.157859.

5. Veronesi U, De Cicco C, Galimberti VE, Fernandez JR, Rotmensz N, Viale $\mathrm{G}$, et al. A comparative study on the value of FDG-PET and sentinel node biopsy to identify occult axillary metastases. Ann Oncol 2007; 18(3):473-8. doi: 10.1093/annonc/mdl425.

6. Fuster D, Duch J, Paredes P, Velasco M, Muñoz M, Santamaría G, et al. Preoperative staging of large primary breast cancer with [18F] fluorodeoxyglucose positron emission tomography/computed tomography compared with conventional imaging procedures. J Clin Oncol 2008; 26(29): 4746-51. doi: 10.1200/JCO.2008.17.1496.

7. Ueda S, Tsuda H, Asakawa H, Omata J, Fukatsu K, Kondo N, et al. Utility of 18F-fluorodeoxyglucose emission tomography/computed tomography fusion imaging $\left({ }^{18} \mathrm{~F}-\mathrm{FDG} \mathrm{PET} / \mathrm{CT}\right)$
C Cancer 2008; 8:165. doi: 10.1186/1471-2407-8-165.

8. Kim J, Lee J, Chang E, Kim S, Suh K, Sul J, et al. Selective sentinel node plus additional nin combination with ultrasonography for axillary staging in primary breast cancer. BMon-sentinel node biopsy based on an FDG-PET/CT scan in early breast cancer patients: A single institutional experience. World J Surg 2009; 33(5):943-9. doi: 10.1007/ s00268-009-9955-z.

9. Heusner TA, Kuemmel S, Hahn S, Koeninger A, Otterbach F, Hamami ME, et al. Diagnostic value of full-dose FDG PET/CT for axillary lymph node staging in breast cancer patients. Eur J Nucl Med Mol Imaging 2009; 36(10):1543-50. doi: 10.1007/s00259-009-1145-6.

10. Jager JJ, Keymeulen K, Beets-Tan RG, Hupperets P, Kroonenburgh MV, Houben R, et al. FDG-PET-CT for the staging of high-risk breast cancer patients reduce the number of further examinations: A pilot study. Acta Oncol 2010; 49(2):185-91. doi: 10.3109/02841860903440262.

11. Cody HSR. Current surgical management of breast cancer. Curr Opin Obstet Gynecol 2002; 14:45-52.

12. Mori M, Fujioka T, Katsuta L, Tsuchiya J, Kubota K, Kasahara $M$, et al. Diagnostic performance of time-of-flight PET/CT for evaluating nodal metastasis of the axilla in breast cancer. Nucl Med Commun 2019; 40(9):958-64. doi: 10.1097/MNM.0000000000001057.

13. Song $\mathrm{BI}$, Kim HW, Won KS. Predictive value of ${ }^{18} \mathrm{~F}-\mathrm{FDG}$ $\mathrm{PET} / \mathrm{CT}$ for axillary lymph node metastasis in invasive ductal breast cancer. Ann Surg Oncol 2017; 24(8):2174-81. doi: 10.1245/s10434-017-5860-0.

14. Carter CL, Allen C, Henson DE. Relation of tumour size, lymph node status, and survival in 24,740 breast cancer cases. Cancer 1989; 63(1):181-7. doi: 10.1002/10970142(19890101)63:1<181:aid-cncr2820630129>3.0.c0;2-h.

15. Aquino RGF, Vasques PHD, Cavalcante DIM, Oliveira ALS, Oliveira BMK, Pinheiro LGP. Invasive ductal carcinoma: Relationship between pathological characteristics and the presence of axillary metastasis in 220 cases. Rev Col Bras Cir 2017; 44(2):163-70. doi: 10.1590/0100-69912017002010.

16. Kutluturk K, Simsek A, Comak A, Gonultas F, Unal B, Kekilli E. Factors affecting the accuracy of ${ }^{18} \mathrm{~F}$-FDG PET/CT in evaluating axillary metastases in invasive breast cancer. Niger J Clin Pract 2019; 22(1):63-8. doi: 10.4103/njcp.njcp_198_18.

17. Porter GJ, Evans AJ, Pinder SE, James JJ, Cornford EC, Burrell $\mathrm{HC}$, et al. Patterns of metastatic breast carcinoma: Influence of tumour histological grade. Clin Radiol 2004; 59(12):1094-8. doi: 10.1016/j.crad.2004.05.001.

18. Sun WY, Choi YJ, Song YJ. Prediction of axillary nodal status according to the axillary lymph node to primary breast tumour maximum standardised uptake value ratio on $18 \mathrm{f}$ fluorodeoxyglucose positron emission tomography/computed tomography. J Breast Dis 2016; 4(2):92-9. doi.org/ 10.14449/jbd.2016.4.2.92.

19. Costa OF Neto, Castro RB, Oliveira CV, Feitosa TVN, Alves JJ Júnior, Cavalcante FP, et al. Predictive factors of axillary metastasis in patients with breast cancer and positive sentinel lymph node biopsy. Rev Col Bras Cir 2017; 44(4):391-6. doi: 10.1590/0100-69912017004014. 
$\bullet \bullet \bullet \bullet \bullet \bullet \bullet \bullet \bullet$ 